\title{
Observation-based Inquiry in a Virtual Ambient Environment
}

\author{
Tom Moher, Andrew Johnson, Yongjoo Cho, Ya-Ju Lin \\ Electronic Visualization Laboratory \\ University of Illinois at Chicago \\ 851 S. Morgan, 1120 SEO (M/C 154), Chicago, IL 60607 \\ Tel: 312.996.4562, Fax: 312.413.7585 \\ Email: moher@eecs.uic.edu
}

\begin{abstract}
Design rationale and user experience are described for a virtual ambient environment designed to support sixth grade students' learning of simple co-occurrence relationships and systematic observational skills. User experience was characterized by enthusiasm, but with significant data loss and navigational difficulties. Design implications and further extensions of virtual ambient environments are suggested.
\end{abstract}

Keywords: Virtual reality, visualization, simulations, scaffolding.

\section{Introduction}

Scientists depend on organized and accurate observations of natural phenomena for the formulation and evaluation of hypotheses; indeed, for some sciences, such as astronomy, observation is everything. The importance of observation as a component of scientific inquiry (Friedler, et al., 1990; Norris, 1985) has led to explicit inclusion of the teaching of observation skills in national science (AAAS, 1993; NRC, 1996) and mathematics (NCTM, 1998) guidelines and standards, with particular emphasis on the importance of providing observation-based activities for young (K-6) learners.

Teachers of young children have always used the real world as the locus for observational activities. From kindergarten students gathering and categorizing fallen leaves to Internet-based projects involving the collection, aggregation, and analysis of global ozone readings, the real world offers authenticity, complexity, and true sensory immersion (Soloway, et al., 1999).

But reality has two important characteristics that may limit its utility in particular learning situations. First, for reasons of distance, time, expense, scale, or safety, real-world environments or tools appropriate to the targeted learning content area might simply be inaccessible. Schools don't have the budgets for field trips to the North Pole or the depths of the Atlantic Ocean, and they don't have the technology to take field trips to Mars, the human circulatory system, or Chicago at the turn of the century. If inquiry grows from the observation of phenomena, the lack of an environment within which one might draw that experience severely limits the range of accessible content domains.

A second, complementary, problem - and our focus in this paper-derives from the very richness of the real world. Even a small slice of the world offers nearly unlimited opportunity for observation. With so many phenomena to choose among, heavy-handed intervention may be needed to channel inquiries in the direction of learning content goals. Moreover, data derived from the real world can be messy, sometimes to the extent that it obscures the very relationships the activity is intended to uncover.

These problems-accessibility and complexity—are often addressed through the use of simulations. Simulations can provide access to otherwise inaccessible phenomena, and simulations afford a great deal of control over which abstractions to feature and the mathematical properties of the data derived from the simulation. Most simulations designed to support inquiry and discovery learning provide users with control over a set of independent variables and access to the resulting impact on one or more dependent variables (e.g., Friedler, et al., 1990; deJong, et al., 1999). Learners literally create the phenomena of interest; the challenge is to provide the scaffolding (Guzdial, 1995) that will help them to plan and conduct a meaningful investigation. 
In this paper, we describe the design and pedagogical context for a different style of simulation, intended to support children's understanding of the earliest phases of scientific inquiry: systematic observation, data collection, aggregation, reduction, and representation, and hypothesis articulation. Our simulations are ambient environments within which users may navigate, observe and record phenomena, and take notes, but may not affect the state of the simulation. These environments reduce control affordances to navigation and visualization, activities which even young learners can understand. We argue that even such simple worlds can be the source of rich and authentic learning experiences for young learners, and are consistent with emerging design frameworks for technologysupported inquiry learning (Edelson, Gordin, \& Pea, 1999). In the balance of this paper, we describe the design of an ambient environment—and concomitant learning unit—which we recently piloted with a sixth grade science class.

\section{The Correlations World}

We have developed an ambient environment designed to support the learning of scientific inquiry skills in the context of introductory concepts of mathematical correlation. In the Correlations World (Figure 1), students collaboratively explore a large "natural" terrain populated with a variety of different plant types. In this environment, some plant types tend to co-occur in clusters with other plant types, while some combinations are never found. By exploring the Correlations World, students discover, represent, and report on the co-occurrence patterns found. The World itself has limited affordances: navigation, the ability to take "snapshots" automatically posted to a class web page, and the ability to plant an unlimited number of (biodegradable) "flags" in the ground. While the terrain (grass, gravel, sand) layout is fixed, the selection and location of plant types is a configurable option. In the Correlations World, the land mass is divided into regions in two independent ways: by the orthogonal arrangement imposed by the picket fences ("sectors"), and by the differential texture maps used on the ground ("terrain").

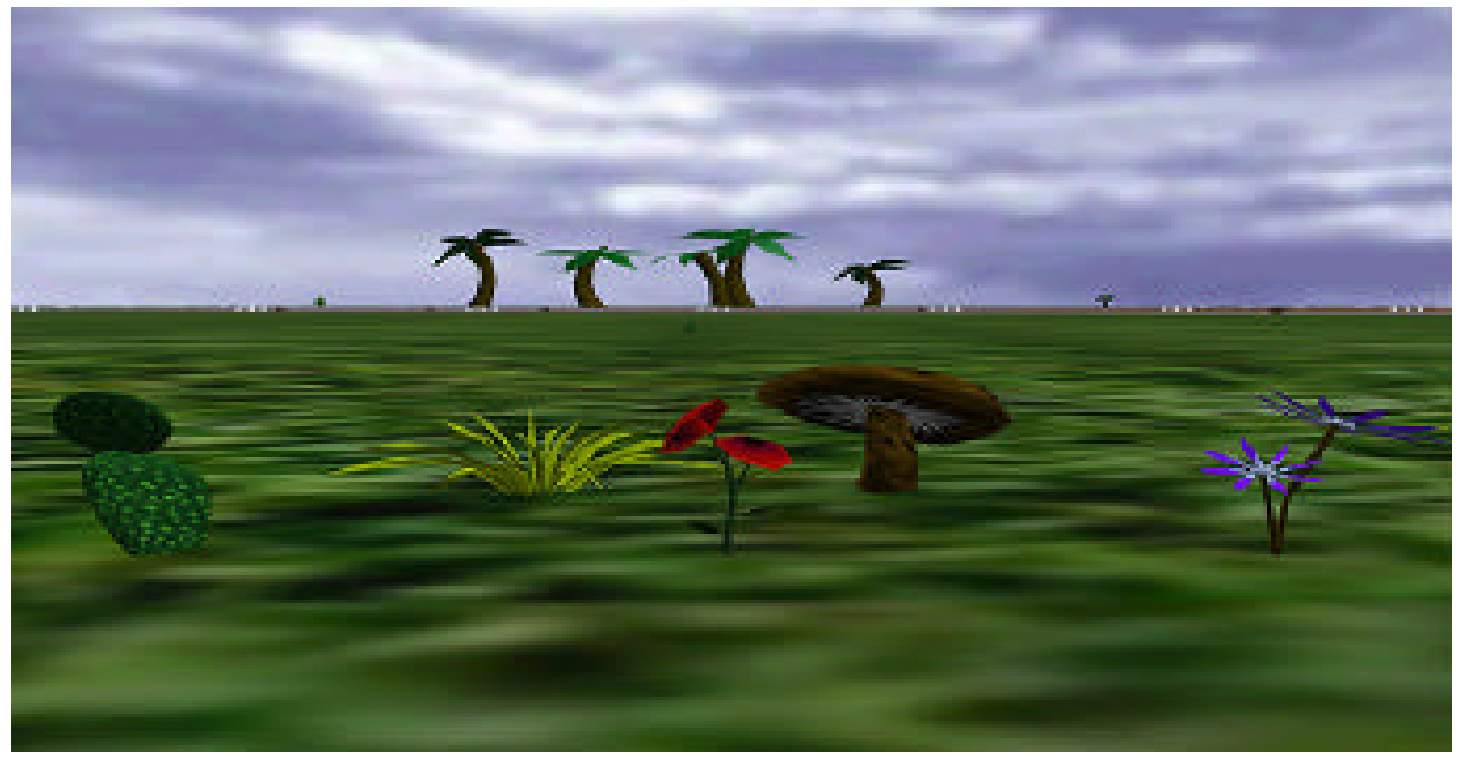

Figure 1. A scene from the Correlations World; countable plants are in the foreground.

The Correlations World has been implemented on an ImmersaDesk ${ }^{\circledR}$, a 50 in. by 67 in. rear-projected video system with head- and hand-tracking, employing lightweight shutter glasses to present a stereoscopic display (Figure 2 ). The decision to implement the synthetic environment on an ImmersaDesk ${ }^{\circledR}$ rather than a desktop display was motivated by the desire to provide a wide visual field-of-view, support collaborative investigations (up to four

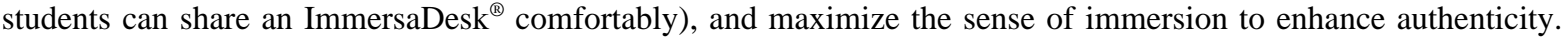
The ImmersaDesk ${ }^{\circledR}$ has been installed on a semi-permanent basis in the Media Center at Lincoln elementary school in Oak Park, Illinois. 


\section{Learning Activities}

For the past few months, we have been working with Lincoln teachers in planning the details of an intervention employing the Correlations World. The unit begins with a brief whole-class introduction conducted by the teacher. The purpose of the introduction is simply to inform the class of the existence of a new environment to be explored, and to select a "scouting team" responsible for a brief initial foray into the environment (with, of course, the concomitant promise that "everyone will get their turn") for the purpose of providing an initial report to the rest of the class on what they found.

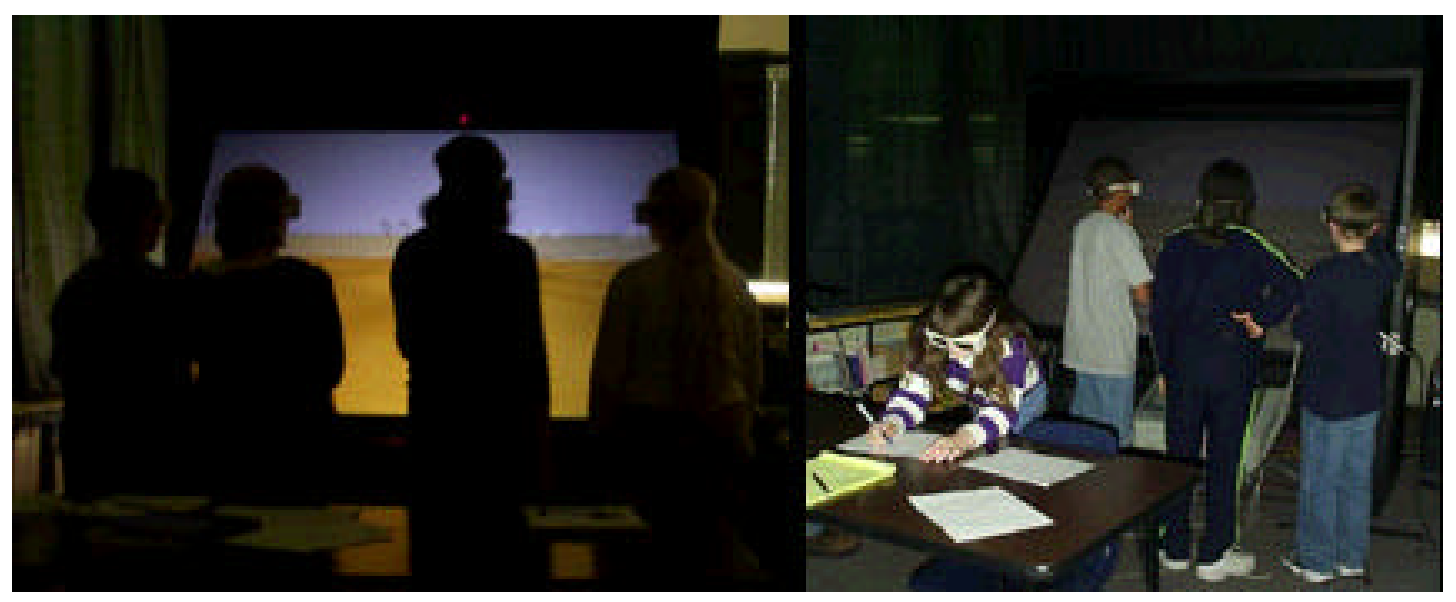

Figure 2. Children exploring the Correlations World at an ImmersaDesk ${ }^{\circledR}$.

During the class's time in the Media Center, the scouting team is "pulled over" to the ImmersaDesk ${ }^{\circledR}$, where they are familiarized with the apparatus and asked to explore the virtual world. In order to support their subsequent report to the class, the team is asked to select a "recorder" who will take (paper and pencil) notes, and are informed that one of the buttons on the navigation controller will allow them to take "snapshots" of the space, which will automatically be saved for them on a web page to show the class; they are encouraged to find other affordances (i.e., the button which plants the flags in the ground).

The teachers are included in the scouting team for the purpose of helping the students attend, at an appropriate level of detail, to the elements of the virtual world. Our experience (Moher, et al., 1999) is similar to that reported by other researchers involved in inquiry learning (Jackson, et al., 1997): it is easy for students to fixate on elements of the simulation which are unassociated with the content learning goal, or, in this case, to focus too tightly on detailed description of particular elements (i.e., low-level details of the plant renderings). The teacher ensures that the scouting team notes (and "photographs") at least the following features of the environment: the presence of different types of plants, the presence of picket fences bounding various regions of the space, the different "ground" types, and the presence of orientation elements (e.g., palm trees, silos, and a rainbow" in the distance corresponding to compass points).

The next phase of the unit takes place back in the classroom. The teacher talks about observation as an important, active component of scientific inquiry, involving more than simply noticing. The scouting team reports both its processes and its findings, hopefully including the observation that the plants grew in clusters, rather than individually, and that some of the clusters contained more than one kind of plant. If these observations are not forthcoming, the teacher might refer other class members to the photographs, and if all else fails, model the observation herself (Tabak \& Reiser, 1999). The teacher finally introduces a question: how could we find the rules about which plants go together in this world?

The ensuing discussion might touch on many subjects (Roth and Bowen 1994). Three issues are of special importance: the nature of the data to be recorded, strategies for coverage (ensuring that the entire space is surveyed), 
and the avoidance of duplicate counting (ensuring that each co-occurrence is counted only once). The outcome of the discussion should be the identification of observation teams and the assignment of teams to particular geographic regions in the Correlations World.

Next comes the actual exploration of the environment by teams. Each team is given approximately 30 minutes to explore their assigned space and to (manually) record co-occurrence groups. Team members are assigned roles (navigator, driver, and recorder) which are rotated during the session.

Once all teams have finished their explorations, activity resumes in the classroom. First, individual teams examine their co-occurrence lists in an attempt to provide a more compact characterization of the rules governing their assigned regions. Finally, individual teams orally report their findings, after which the class undertakes a process of data aggregation and attempts to produce rules capable of characterizing the entire Correlations world.

\section{Classroom Experience}

In January, 2000, the first pilot study of the Correlations World project was completed. Two combined sixth grade classes (46 students total) participated in the unit, over the course of one week. All of the activities — both in class and at the ImmersaDesk ${ }^{\circledR}$ - were video- and audio-recorded. Eight distinct plant types were used in the pilot, with approximately 400 plants distributed over the landscape. Plants occurred in "patches" of approximately 6-10 plants apiece; each patch contained exactly two types of plants. Among the 28 different possible co-occurrence pairs, 16 were actually deployed, in two disjoint groups. Twelve of the pairs were distributed among seven of the "sectors" bounded by the fences, with each sector containing $6 \pm 1$ unique pairs. The remaining two sectors contained four different co-occurrence pairs, with three unique pairs placed in each sector. Some sectors contained multiple occurrences of the same co-occurrence pair.

It was no surprise that the unit was well-received by the students; for most of them, this was their first experience with a VR environment, and the novelty of that environment, along with the presence of the researchers and recording equipment, generated a great deal of interest and excitement. This novelty effect confounds the assessment of the degree to which the children are engaged by the unit; indeed, overcoming the novelty effect is one of our long-term goals in establishing a semi-permanent facility at Lincoln.

Due to the large class size, two scouting teams of four students apiece performed the initial exploration of the Correlations World. Each group spent approximately 30 minutes at the ImmersaDesk ${ }^{\circledR}$, with navigation control shared among participants. Navigational control was provided by a "wand" consisting of an isometric trackball and three button switches, and was quickly mastered by the students.

A significant amount of ad hoc scaffolding was required during the scouting sessions. Teams had to be reminded frequently to take "photographs" of the space for their subsequent presentation to the whole class; in the end, each group took about 10 pictures. The navigational strategies used by the teams were decidedly random. While we were careful to avoid pre-specifying the "interesting" features of the environment during scouting, intervention was required to ensure that the students did not spend all of their time exploring "ancillary" features at the expense of the recognizing the presence of the "patches" and the consistency of the "two plant types per patch" rule. Visual elements designed to provide navigational orientation, such as the rainbow, were of great interest to the children; however, they were placed at such extreme distances that allowing the children to navigate to one of their positions would have required almost 15 minutes at full speed. While paper and pencil were provided for the students, and they were encouraged to take notes, they were not required to do so, and in fact no written notes were taken by the students during the initial explorations. Only one of the groups happened upon the mechanism for placing the "biodegradable flags" (pushing the rightmost button on the wand). The scouting groups were subsequently given a short amount of time (about 15 minutes) to plan their presentations to the class, but were given no explicit directions or tools to support that planning.

The class discussion following the scouting activities lasted approximately one hour. The presentations by the scouting teams lasted about 30 minutes. While the presentations were somewhat haphazard, they did include an explicit articulation of the "two plant types per patch" phenomenon (among many other observations). The remaining students in the class were full of questions for the scouting team; indeed, we were finally forced to cut off questions in order to allow time for planning the subsequent activities. 
Because the students did not spontaneously settle on the "co-occurrence problem" as a driving question, we were forced to explicitly introduce the task of developing a systematic survey of that phenomenon as their charge. When prompted, the scouting teams agreed that the task would be time-consuming; one girl estimated (with surprising accuracy) that a survey of the entire space would take about four hours. The class was provided with a map of the Correlations World, which clearly depicted the nine sectors, overlaid on a texture map representing the distinctive terrain types. After some discussion, they decided to split into teams of four students apiece, with each group assigned to one of the sectors bounded by the fences. The teacher told the class that she would assign individual students to teams.

The remainder of the available time focused on three problems, raised by the teacher. First, the students were asked how they could ensure that they didn't accidentally count the same "patch" twice. A girl from one of the scouting parties offered the suggestion that the teams leave a flag near each patch which they counted; this suggestion met general approval from the class, and was adopted as part of their standard strategy. Next, the teacher asked the students how they could be sure that they counted every patch in their assigned sector. There was consensus among the scouting parties that seeing the patches within a sector required navigation; they were not all immediately visible to the "naked eye." One girl suggested that a strategy similar to "mowing a lawn"; again, the class was in general agreement that this would be effective. Finally, the teacher asked the students exactly what data they would record when they encountered a patch. The students decided that they would record the two types of plants found in each patch. One boy followed up on this decision by suggesting that the terrain type might be related to the types of plants found; after some discussion, the class decided to also record, for each patch, the type of terrain on which it had been found.

On the following two days, students were pulled out of class by team and given approximately 30 minutes to explore their (chosen) sector. Each team was given a brief introduction to the apparatus, reminded of the data which they had decided as a class to collect, and were given assistance in navigating to their assigned sector. During the data collection process itself, the students operated largely independently; the researchers intervened only to (1) remind the students to record data, (2) ensure that navigation control was shared during the session, (3) prevent teams from going outside the bounds of their assigned sector, and (4) remind the students to collect sufficient information about the plants (color, shape) to ensure a common vocabulary upon completion. (This was necessary because the students had not had time to develop shared names for the plant types during the initial discussion, and because the scouting teams had not found all of the unique plant types during their initial explorations.)

Data collection was inconsistent. Only two of the nine groups actually managed to find all of the patches contained in their sector. While the flags were used consistently (and effectively) to avoid double counting, not a single group (even the successful ones) employed a systematic algorithm for ensuring that they had found all of their patches. Responsibility for data collection was not assigned to individual students, but left to the group, and given the high level of engagement with the exploration itself, students were reluctant to assume that responsibility. As a consequence, some patches that were encountered were never recorded. Only four of the nine groups remembered to record the terrain type (as they had agreed to do in class).

Following data collection, the class met as a whole for an hour to discuss their findings. In an effort to settle upon a common vocabulary, the teacher again showed the class the pictures which the scouts had taken, and asked the class to compile a list of plant names. The class negotiated standard names for the plants which the scouts had found, and then identified two additional plant types which had been found only during the actual survey. Volunteers drew pictures on the blackboard of the new types, with vociferous input from other class members.

Next, the teacher drew a $3 \times 3$ grid on the blackboard and asked each team to write the results of their surveyed section in the appropriate cell. Because space was limited, the children settled on a strategy of using a single letter to represent each plant type (serendipitously, all of their chosen names began with a different letter of the alphabet). (An examination of the raw data sheets indicated that several pairs were lost during this transcription process.) The students were then asked whether they could see any patterns in the way that the co-occurrence pairs were distributed. They noted the presence of some common pairs across cells, as well as the non-uniformity of the distribution of patches, but were otherwise unable to make much progress.

The teacher asked them whether every type of plant occurred in a patch with every other type of plant; the students were confident that this was not the case. The teacher then asked them how many possible different types of 
pairs there could be. After several random guesses were put forward, the teacher drew an $8 \times 8$ (co-occurrence) grid on the board, labeled the rows and columns by the plant types, and asked the class for a possible interpretation of each cell. One girl put forward the idea that each cell could be used to indicate whether that kind of (row,column) pair had been found. The students immediately offered the observation that they didn't need the diagonal of the $8 \times 8$ grid, since all of the patches contained two different types of plants. There quickly followed some confusion related to the unordered nature of the pairs, and the children recognized that only a triangular portion of the $8 \times 8$ grid was desirable. Upon counting, the students discovered that there were 28 possible co-occurrence pairs.

There followed an enthusiastic suggestion that we could systematically convert the data from our $3 \times 3$ (geographic) map to the triangular (co-occurrence) grid, putting a mark in a cell for each pair contained in the map. A boy suggested that instead of a mark, we should indicate the number of the map sector in which the pair was found. Each team in turn converted their data to the new representation on the blackboard. About $10 \%$ of the data were initially lost during this process; however, since the rest of the class now had access to the initial geographic data, they vociferously corrected their classmates' errors of transcription.

A second, 30-minute session was held to try to discuss the aggregated data. The students focused most of their attention on the co-occurrence matrix. In spite of the data loss, the redundancy of plant distribution allowed them to find 14 of the 16 pairs. The students noted that some plants seemed to grow "next to almost any other kind," while some plants were contained in only a few type pairs. The students did not spontaneously report the fact that two of the sectors co-occurrence pairs were disjoint from those found in the other sectors. However, upon a query from the teacher, "are any of these sectors different from the others?," several students noted that the sectors 1 and 7 (the two special sectors) were either found alone in the cells of the co-occurrence matrix, or in combination with each other, but that sectors 1 and 7 were never in a co-occurrence cell with the other sector numbers. There followed a brief discussion of how to represent the aggregated findings: it was decided that instead of articulating a single rule set, they would produce two sets of rules: one for sectors $1 \& 7$, the other for the remaining sectors.

Finally, we asked the students whether they were confident that they had found all of the patches. In turn, each group expressed absolute confidence that they had successfully found all of their plants. However, when asked as a class whether the whole class had found all of the plants, there was split opinion. It was decided that a small "follow up" group would go back into the space to do some spot-checking. That group re-surveyed three of the sectors, and found (correctly) that two of the three had been counted incorrectly. Interestingly, the "follow up" team did make a strong (though not always successful) attempt to use the "lawn mower" algorithm to ensure coverage. They reported their findings to the class.

While the Correlations unit was received with enthusiasm by the sixth grade teacher and students, a great deal of scaffolding was required to help the students find their way through the learning unit:

Direction control. The isometric trackball was very useful for turning, but it is difficult to effect straight forward motion without weaving left and right. In the next version of the Correlations World, the user will be able to use the trackball as in the initial version, but will also be able to press the middle button for full-speed forward motion.

Navigation. Students had a great deal of difficulty settling on a systematic navigational strategy, and finding and staying within their sectors. We will improve the visibility of sector boundaries, and will experiment with the provision of a bird's-eye map (including current position), displayed on a laptop computer display adjacent to the Immersadesk ${ }^{\circledR}$.

Data integrity. Data were lost at every stage of the process. Teams found patches and forgot to record them. They used names and recording techniques which led to the recording of ambiguous data. They failed to copy their group's data to the aggregate geographic table. They failed to correctly copy from the geographic table to the co-occurrence matrix. In the next version of the Correlations project, we will provide data collection forms, we will allocate time explicitly for class discussion of shared terminology, and we will extend the scouts' "snapshot" mechanism to all teams (to support the recording of new plant types). 
Perhaps the main result was that we had significantly underestimated the amount of time which would be required for in-class activities; easily twice as much time could be profitably allocated. In future interventions, the in-class time period will be substantially increased.

\section{Variations}

By varying the number and arrangement of plants in the Correlations World, it is possible to reuse the system to explore a broad range of increasingly sophisticated issues in correlation and scientific exploration. In the simplest case, we can employ uniform co-occurrence rules across the entire space, so that the final aggregation activity serves only to extend and confirm team observations.

The data set we employed with the Lincoln sixth grade varied the co-occurrence rules based on an external variable: rectangular sector. Assuming that students choose to assign teams to sectors for the exploration activity, we can introduce complexity into the data aggregation phase of the unit by introducing differential co-occurrence rules among those sectors. Instead of finding that the findings of individual groups are confirmed or extended by the other groups' findings, conflicting rule sets will arise, leading to the need for the students to represent and report their aggregate findings in a new way.

Varying co-occurrence rules by terrain type leads to a different problem. If students organized teams by sector, they may discover discrepancies even within their assigned area of exploration. (In the current texture map, some sectors have a uniform terrain, while others contain up to three terrain types within a single sector.) Some groups will find a coherent set of co-occurrence rules; others may conclude that the rules are inconsistent, or even non-existent. An ensuing discussion might lead to two important conclusions: that the assignment of teams to sectors was the wrong way to organize activity, or that, in retrospect, it was necessary to collect an additional piece of data during exploration (i.e., terrain type). In either case, the result will be that the students have to undertake a second survey, using different methods.

A different problem arises when the co-occurrence relations between pairs of plants are governed not by an external variable, but by the presence or absence of a third plant type. For example, one plant type might be assigned the role of a triplet inhibitor; that is, it might co-occur with other types of plants on a pairwise basis, but preclude the co-occurrence of otherwise "friendly" plants by its presence. The possibilities here are almost arbitrarily complex, providing opportunities for the re-use of the environment at more advanced grade levels, or for providing activities with differential levels of challenge within heterogeneous classrooms.

By introducing a single empirical exception to an otherwise consistent set of co-occurrence rules, we raise the opportunity for an interesting discussion of the differences between mathematical proof and scientific evidence. The plants can be arranged so that, for example, there are dozens of instances of each co-occurrence pairs, except in a single case, where there is only a single co-occurrence, discovered by only one group. Does this invalidate the other co-occurrence rules? How should it be reported; does frequency matter?

The addition of a confirmation phase to the learning unit (e.g., by leaving one sector uninvestigated until the rules have been discovered) further extends the learners' introduction to scientific inquiry. In the early grades, confirmation may simply be motivated as a necessary step for "checking your work," an important component of scientific investigations in its own right. By varying co-occurrence rules in the confirmation phase in later grades, we gain the opportunity to discuss the important concept of the generality of scientific findings.

\section{Beyond Correlations}

The ambient environment paradigm easily extends to content areas beyond the early stages of mathematical correlation. The aforementioned designs are predicated on the assumption that the entire population under consideration can be observed. Alternatively, by limiting the time allocated for initial exploration, we could introduce the notion of population sampling. Then, by conducting a second, complete survey, students could discuss the power and limitations of sampling, noting both time efficiency relative to surveying a complete population, and the inaccuracies introduced by failing to discover the complete co-occurrence rules.

Replacing the flora with fauna opens the way for a whole new style of exploration, and raises questions about our exploration methods ("The animals are moving; do we sit still and watch, or do we move around?"), 
coverage ("How do we know we've found all the animals in the environment?"), and preventing duplicate counting ("With the plants, we left flags to indicate they were counted; what do we do with animals that move around?").

Even simpler worlds might focus more directly on the acquisition of skill in the detailed observation of individual objects or the classification of objects based on similar or dissimilar features. Marginally richer (but still simple) worlds could equip students with transducers (e.g., thermometers, rules, scales), to extend their powers of observation beyond those available to the naked eye.

\section{References}

American Association for the Advancement of Science (AAAS) (1993). Benchmarks for Science Literacy: Project 2061. New York, NY: Oxford University Press.

Edelson, D., Gordin, D., and Pea, R. (1999). Addressing the Challenges of Inquiry-Based Learning through Technology and Curriculum Design. Journal of the Learning Sciences, 8, 391-450.

Friedler, Y., Nachmia, R, and Linn, M. (1990). Learning scientific reasoning skills in microcomputer-based laboratories. Journal of Research in Science Teaching, 27, 173-191.

Guzdial, M. (1995). Software-Realized Scaffolding to Facilitate Programming for Science Learning. Interactive Learning Environments, 4, 1-44.

de Jong, T., Martin, E., Zamarro, J., Esquembre, F., Swaak, J., and van Joolingen, W. (1999). The Integration of Computer Simulation and Learning Support: An Example from the Physics Domain of Collisions. Journal of Research in Science Teaching, 36, 597-615.

Moher, T., Johnson, A., Ohlsson, S., Gillingham, M. (1999). Bridging Strategies for VR-Based Learning. ACM Conference on Human Factors in Computing Systems (CHI '99). Pittsburgh, PA, May 15-20, 1999, 536543.

National Council of Teachers of Mathematics (NCTM) (1998). Principles and Standards for School Mathematics Electronic Version: Discussion Draft (http://standards-e.nctm.org/1.0/normal/index.html).

National Research Council (NRC) (1996). National Science Education Standards. Washington, D.C.: National Academy Press.

Norris, S. (1985). The philosophical basis of observation in science and science education. Journal of Research in Science Teaching, 22, 817-833.

Roth, W-M. and Bowen, G. M. (1994). Mathematization of Experience in Grade 8 Open-Inquiry Environment: An Introduction to the Representational Practices of Science. Journal of Research in Science Teaching, 31, 293-318.

Soloway, E., Grant, W., Tinker, R., Roschelle, J., Mills, M., Resnick, M., Berg, R., and Eisenberg, M. (1999). Science in the Palms of Their Hands. Communications of the ACM, 42, 21-26.

Tabak, I., \& Reiser, B. J. (1999). Activity Attributes: Steering The Course of Dialogue in Inquiry-Based Science Classrooms. Presented at 1999 Annual Meeting of the American Educational Research Association, Montreal, Canada.

\section{Acknowledgments}

We wish to express our appreciation to Marilyn Rothstein, Betty Smitherman, Carol Dudzik, and the sixth grade students at Lincoln school. Thanks also to Jason Leigh for technical support and design assistance. This research was made possible through major funding from the National Science Foundation, specifically EIA9802090, EIA-9720351, and DUE-9979537.

The ImmersaDesk is a trademark of the Board of Trustees of the University of Illinois. 